\title{
Incidence of Incidental Thyroid Nodules on Computed Tomography (CT) Scan of the Chest Performed for Reasons Other than Thyroid Disease
}

\author{
Kurt Scherer, Seth Means, Collins Chijioke, Paul Karmin, Mukta Panda \\ Department of Radiology, Transitional Year Program and Department of Internal Medicine, College of Medicine, University of \\ Tennessee, Chattanooga, USA. \\ Email: kscherer5@gmail.com
}

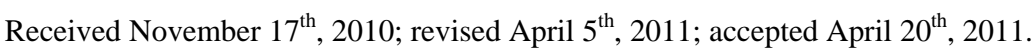

\begin{abstract}
Previous studies demonstrate an approximately 16\% incidence of incidental thyroid nodules (ITNs) on Computed Tomography (CT) of the head/neck and thorax combined. Malignant disease is present in many cases. No study to date has quantified ITNs on CT of the thorax alone, an examination performed more frequently than head/neck CT. Our objective was to determine the prevalence and significance of ITNs with further stratification based on size criteria of non-dominant $(<10 \mathrm{~mm})$ and dominant $(>10 \mathrm{~mm})$ on CT imaging of the chest performed for indications other than thyroid disease and to assess if these were further evaluated. A retrospective analysisof 500 patients (257 men and 243 women; mean age, 58.3 +/- 16.7 years) with thoracic CT scans performed at Erlanger Health System from October 2007 to October 2008 was performed. ITNs were noted in 61 (12.2\%) of patients. Nodules were solitary in 43 patients (70.5\%) and multiple in 18 (29.5\%). Thirty-three patients (6.6\%) were found to have a dominant nodule $>10 \mathrm{~mm}$. Sixteen of the 33 patients with dominant nodules received further workup showing benign pathology in 50\%. More than $50 \%$ of the 33 dominant nodules received no follow-up at all. Chest CT demonstrates many ITNs. The incidence in this study was $12.2 \%$ with $6.6 \%$ being potentially malignant dominant thyroid nodules. Partial thyroid glands were visualized in 58.2\% exams, implying an incidence of $12.2 \%$ ITNs is an underestimate. It may be beneficial for routine chest CT to be extended $2 \mathrm{~cm}$ superiorly in order to ensure full visualization of the thyroid gland and related pathology.
\end{abstract}

Keywords: Incidental Thyroid Nodules, Thyroid Malignancy

\section{Introduction}

Thyroid abnormalities, specifically thyroid nodules are routinely identified on CT imaging. To date, studies have shown that incidental findings of the thyroid occur at the rate of $16 \%$ on CT imaging and $27 \%$ on sonography $[1,2]$. However, the incidence of these nodules has only been studied with imaging of the head and neck [3]. The frequency of chest imaging is increasing. Wittram, et al. report a significant increase over a decade (with $P$ $=0.04$ for inpatient and $P=0.01$ for outpatients) [4]. With this increase, there actually may be an increase in the previously reported incidence of these abnormalities.

Furthermore, the indications for chest CT are much wider than for head and neck CT scans, which frequently include chest CT solely for detection of metastases in malignant disease of the head and neck. The true incidence of thyroid disease in CT chest exams with no head and neck malignancy is unknown. In patients with a known malignancy other than thyroid, incidental thyroid nodules were found to be malignant in $24 \%$, which is above the expected rate of $5 \%$ in traditionally discovered nodules [5]. From the reported data, it was unclear whether these incidental findings were initially made on head and neck CT or chest CT alone [5].

In general it has been reported that among incidentally detected lesions of the thyroid gland, there was at least a $3.9 \%$ rate of malignancy and $7.4 \%$ rate of malignant potential, with at least an $11.3 \%$ prevalence of malignant or potentially malignant lesions among incidental thyroid abnormalities detected on CT [6]. The overall increased use of CT chest may demonstrate more thyroid nodules to be managed and subsequently require that all CT chest scans include the thyroid gland as a means of coincidental screening for thyroid disease, benign and 
malignant which has potential clinical significance.

In summary, previous studies have shown an approximately $16 \%$ incidence of incidental thyroid nodules on CT scan of the head/neck and thorax combined. However, no study to date has been performed to identify incidental thyroid nodules on CT scan of the thorax alone.

We conducted a retrospective study to evaluate 500 CT scans of the chest performed at Erlanger Health System to first determine the incidence of incidental thyroid nodules (non-dominant $5-10 \mathrm{~mm}$ and dominant $>10 \mathrm{~mm}$ ) on CT scan of the chest done for indications other than thyroid disease and second, to determine whether those subjects with incidental thyroid nodule were further evaluated with thyroid function testing, ultrasonography, fine needle aspiration, and/or nuclear medicine scintigraphy. We will comment as to whether or not incidental nodules are considered significant, i.e. pursued with further evaluation. This will be included in our final discussion.

Our hypothesis is that with the increased use of CT scans of the chest, the incidence of incidental thyroid nodules found will be higher than previously reported on CT head/neck imaging.

\section{Methods and Design}

500 CT scans of the chest (both contrast and non-contrast) performed at Erlanger Health Science Center for reasons other than thyroid pathology were reviewed retrospectively to identify thyroid nodules. These CT Scans were performed from October 2007 to October 2008 and selected from the PACS. For patients with positive nodules on the CT scans, medical records were reviewed to document if further evaluation (thyroid function testing, ultrasound, radioactive iodine uptake, nuclear medicine, and/or scintigraphy) was performed for positive nodules.

CT scans of patients with predetermined and documented thyroid pathology in their medical records were excluded. The study refers to incidental thyroid nodules, with non-dominant nodules measuring less than $10 \mathrm{~mm}$ and dominant nodules measuring greater than $10 \mathrm{~mm}$. This distinction was performed because of a suspected higher malignant potential of a dominant nodule. No patient identification was recorded during image viewing and comparison with medical record. Data was identified as a numerical number, 1 - 500 on an excel spreadsheet.

All evaluators were initially trained by a board certified Radiologist regarding the CT criteria for thyroid nodules. Also, all evaluators reviewed the anatomy of the thyroid region with special attention to imaging through Brant \& Helms Diagnostic Imaging. After in- dependent data collection and analyses by the individual evaluators, all the positive and questionable negative findings were reviewed collectively by the evaluators in order to address inter-observer variability.

The supervising radiology faculty did a final read to confirm the positive findings and review any questionable negatives. Using Statistical Analysis System (SAS) software, prevalence, frequency, and cross-tabulation data were determined. Data was further categorized by age, gender and race, size, location and number of nodules, +/- contrast, further evaluation of positive nodules, and negligible, partial, or complete imaging of the thyroid.

The inclusion criteria were 500 CT scans of the chest or including the chest, for non-thyroid etiologies, including scans with no thyroid, partial thyroid, or full thyroid. The exclusion criteria were 1) CT scans performed for suspected thyroid disease as indicated on ordering diagnosis; 2) CT scan performed in patient with known thyroid disease if indicated on order; 3) CT scan of inadequate quality for thyroid gland evaluation; 4) Repeat studies of same patient; 5) High resolution CT, as they tend to be exams of the lung parenchyma.

The criteria for positive thyroid nodule identification are as detailed below:

1) $<0.5 \mathrm{~cm}$ (5 mm) incidental thyroid nodule is considered non viable in this study.

2) $0.5 \mathrm{~cm}-1.0 \mathrm{~cm}(5 \mathrm{~mm}-10 \mathrm{~mm})$ incidental thyroid nodule is included as a non-dominant nodule

3) $>1.0 \mathrm{~cm}(10 \mathrm{~mm})$ incidental thyroid nodule is included as a dominant nodule

\section{Result}

500 CT scans of the chest were reviewed in our study. The frequency of contrast use was 254 (50.8\%) compared to non-contrast study 246 (49.2\%). The age range was 18 and 93 with mean of 58.3. Mean number of nodules 1.7 and mean nodule size of $13.7 \mathrm{~mm}$. The malefemale ration in this study was 257:243.

Sixty-one (12.2\%) of 500 had ITNs (Table 1 and Figure 1(a)-(d)), of which 28 were males and 33 females. The abnormality was solitary in 43 patients $(70.5 \%)$ and multiple in 18 (29.5\%). Of the 61 cases with thyroid nodules, 33 (54.1\%) were dominant thyroid nodules measuring greater than $1 \mathrm{~cm}$ (Table 2). Of the 500 total CT scans, 10 did not include the thyroid gland, 291 (58.2\%) included partial thyroid gland and only 199 (39.8\%) included the full thyroid image (Table 3). The majority of the nodules were found in the CTs with partial thyroid image vs. full thyroid image 37 (60.7\%) vs. 24 (39.3\%) (Table 4). Furthermore 19 of the 33 (57.6\%) dominant thyroid nodules were found in the CT scans 


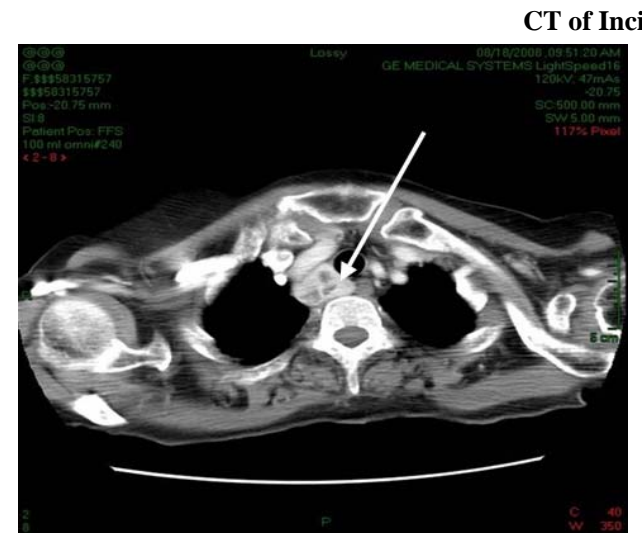

(a)

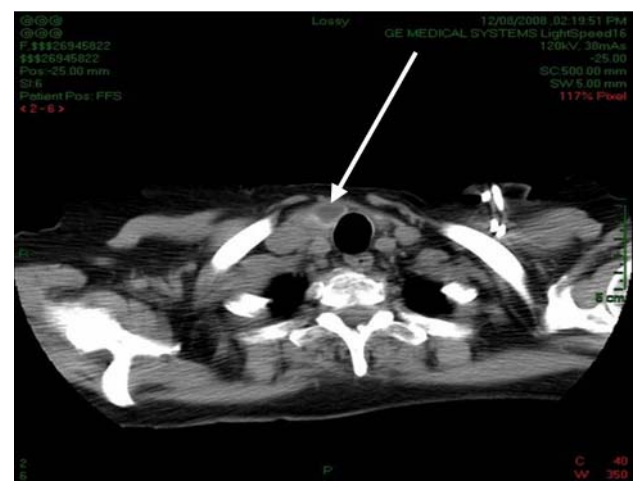

(c)

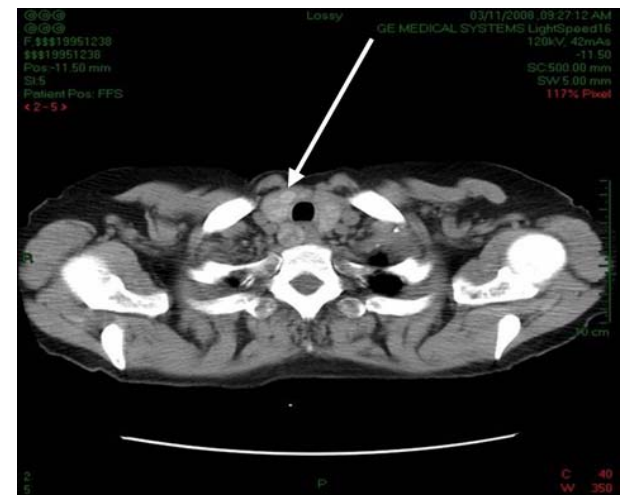

(b)

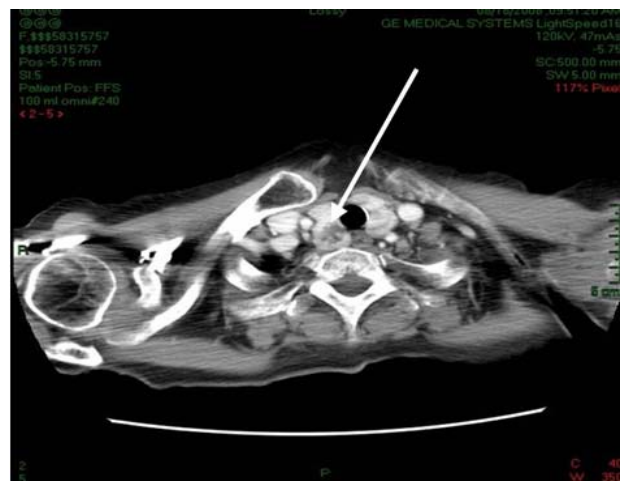

(d)

Figure 1. (a)-(d): Examples of incidental thyroid nodules on CT thorax.

Table 1. Thyroid nodule frequency $(n=500)$.

\begin{tabular}{lcc}
\hline & Frequency & Percent \\
\hline No thyroid nodule identified & 439 & 87.8 \\
Thyroid nodule identified & 61 & 12.2 \\
\hline
\end{tabular}

Table 2. Type of nodule by size $(n=500)$.

\begin{tabular}{lcc}
\hline & Frequency & Percent \\
\hline$<5 \mathrm{~mm}$ & 9 & 1.8 \\
Non-dominant $(5-10 \mathrm{~mm})$ & 19 & 3.8 \\
Dominant Nodule $(>10 \mathrm{~mm})$ & 33 & 6.6 \\
\hline
\end{tabular}

Table 3. Negligible, partial and full thyroid gland imaged ( $n$ = 500).

\begin{tabular}{lccc}
\hline & Frequency & Percent \\
\hline Negligible Thyroid Gland Imaged & 10 & 2.0 \\
Partial Thyroid Gland Imaged & 291 & 58.2 \\
Full Thyroid Gland Imaged & 199 & 39.8 \\
\hline
\end{tabular}

with partial thyroid image whereas 14 (42.4\%) were found in those with full thyroid image (Table 5).

Only 26 of the $61(42.6 \%)$ received additional studies of their thyroid nodules and the remainder did not additional information documented in the medical record (Table 6). Sixteen of the 33 (48.4\%) patients with domi-
Table 4. Number of images with partial vs. full thyroid gland and number of thyroid nodules identified $(n=500)$.

\begin{tabular}{lcccc}
\hline & $\begin{array}{c}\text { Negligible } \\
\text { Thyroid Im- } \\
\text { aged }\end{array}$ & $\begin{array}{c}\text { Partial Thyroid } \\
\text { Imaged }\end{array}$ & $\begin{array}{c}\text { Full Thyroid } \\
\text { Imaged }\end{array}$ & Total \\
\cline { 1 - 2 } $\begin{array}{l}\text { No Thyroid } \\
\begin{array}{l}\text { Nodule } \\
\text { Thyroid Nodule }\end{array}\end{array}$ & 10 & 254 & 175 & 439 \\
$\begin{array}{l}\text { Identified } \\
\text { Total }\end{array}$ & 0 & 37 & 24 & 61 \\
\hline
\end{tabular}

nant nodules received further workup, consiting of thyroid function tests, sonography, or nuclear medicine scintigraphy (Table 7). Six of the 16 (37.5\%) patients were found to have non-toxic multinodular goiters, one Hashimoto's thyroiditis, one colloid cyst, and no final diagnoses in the remaining eight.

\section{Discussion}

CT scan has become a common procedure, especially in the Emergency Department where most patients with pulmonary and cardiac symptoms routinely obtain one. With the increasing frequency of this test, we had initially postulated an increase in the incidence of ITNs. 
Table 5. Negligible, partial and full thyroid gland imaged classified by thyroid nodule sizes $(n=500)$.

\begin{tabular}{lcccc}
\hline & Negligible Thyroid Gland Imaged & Partial Thyroid Gland Imaged & Full Thyroid Gland Imaged & Total \\
\hline$<5 \mathrm{~mm}$ & 0 & 5 & 4 & 9 \\
Non-dominant $(5-10 \mathrm{~mm})$ & 0 & 13 & 14 & 19 \\
Dominant Nodule & 0 & 19 & 175 & 33 \\
(>10 mm) & 10 & 254 & 199 & 439 \\
No Nodule Identified & 10 & 291 & 500 \\
Total & &
\end{tabular}

Table 6. Additional studies on cases with thyroid nodule identified $(n=61)$.

\begin{tabular}{lcc}
\hline & Frequency & Percent \\
\hline $\begin{array}{l}\text { No studies noted in medical record } \\
\text { Presence of additional FU (TFT, U/S, RAIU, }\end{array}$ & 26 & 7.0 \\
NucMed, others) & 26.2 \\
\hline
\end{tabular}

FU: follow up; TFT: thyroid funtion test; U/S: Ultrasonography; RAIU: Radioactive Iodine Uptake Test; NucMed: Nuclear medicine.

Table 7. Description of types of additional studies performed $(n=26)$.

\begin{tabular}{lcc}
\hline & $\begin{array}{c}\text { No studies noted } \\
\text { in medical record }\end{array}$ & $\begin{array}{l}\text { Presence of additional FU } \\
\text { (TFT, U/S, RAIU, NucMed, } \\
\text { others) }\end{array}$ \\
\hline$<5 \mathrm{~mm}$ & 8 & 1 \\
$\begin{array}{l}\text { Non-dominant } \\
(5-10 \mathrm{~mm})\end{array}$ & 10 & 9 \\
$\begin{array}{l}\text { Dominant Nodule } \\
(>10 \mathrm{~mm})\end{array}$ & 17 & 16 \\
\hline
\end{tabular}

The current literature evaluating the incidence of ITNs found on routine CT scans is paucity.

The previously reported incidence of ITNs is $16 \%$ [2, 3]. As has been previously reported, there is an $11.3 \%$ prevalence of malignant or potential malignant lesions among incidental thyroid abnormalities detected on CT scans [6]. In our study we found similar percentages. The incidence of ITNs in our study is $12.2 \%$ with more than $50 \%$ being dominant nodules. About fifty percent received further evaluation of the nodules. To our knowledge, there are no guidelines for further evaluation of incidental nodules found on routine CT scans.

This study indicated that less than $50 \%$ of the 61 cases of ITNs had the full thyroid gland imaged. Given this fact, we suggest that it may be beneficial for CT scans of the chest to be extended superiorly $2 \mathrm{~cm}$ in order to visualize more of the thyroid gland and thus increase the frequency of detecting incidental thyroid pathology without exposing the patients to unnecessary amount of additional radiation.

Though this study is a single center, retrospective, observational, dependent on accuracy of orders for CTs and convenience sample, it still, however, identified some relatively simple system changes that may assist in early identification and follow up of thyroid nodules especially in high risk patients. We recommend a future prospective study to further evaluate ITNs by obtaining history of previous malignancy.

The overall increased use of CT chest may demonstrate more thyroid nodules to be managed and subsequently require that all CT chest scans include the thyroid gland as a means of coincidental screening for thyroid disease, benign and malignant which has potential clinical significance

The study also suggested that the use of contrast does not improve the detection of thyroid nodules and hence may not be use in chest CTs except when indicated for other reasons.

This study serves as a pilot for future study to further evaluate the incidence and consequence of ITNs that may assist to provide standardized guidelines for clinicians in facilitating further management of ITNs.

The strengths of the study include detailed exclusion criteria, limited variability as all scans were obtained from the same hospital and CT scanners, limited variability as all scans were evaluated on the same PACS system and by evaluators with similar thyroid nodule criteria training.

The limitiations of the study include a single center study, retrospective study, observational study, dependency on accuracy of orders for CTs, and convenience sample.

\section{Conclusions}

Given the separate findings including that the incidence of ITNs in our study is $12.2 \%$ with more than $50 \%$ being dominant nodules, the previously described $11.3 \%$ malignant potential of these incidental thyroid nodules, and the fact that $50 \%$ of the 61 cases of ITNs did not receive complete visualization of the thyroid gland on crosssectional imaging, we recommend extending superiorly by $2 \mathrm{~cm}$ the extent of the CT scans of the thorax in order to visualize more of the thyroid gland and thus increase the frequency of detecting incidental thyroid pathology without exposing the patients to unnecessary amount of additional radiation.

\section{REFERENCES}

[1] A. Brander, P. Viikinkoski, J. Nickels and L. Kivisaari, 
“Thyroid Gland: US Screening in a Random Adult Population,” Radiology, Vol. 181, No. 3, 1991, pp. 683-687.

[2] D. M. Yousem, T. Huang, L. A. Loevner and C. P. Langlotz, "Clinical and Economic Impact of Incidental Thyroid Lesions Found with CT and MR,” American Journal of Neuroradiology, Vol. 18, No. 8, 1997, pp. 1423-1428.

[3] Y. S. Rho and H. Y. Ahn, "The Prevalence and Significance of Incidental Thyroid Nodules Identified on Computed Tomography," Journal of Computer Assisted Tomograph, Vol. 32, No. 5, 2008, pp. 810-815. doi:10.1097/RCT.0b013e318157fd38

[4] Wittram, M. B. Conrad, J. Margaret, et al., "Trends in Thoracic Radiology Over Decade at a Large Academic Medical Center,” Journal of Thoracic Imaging, Vol. 19,
No. 3, 2004, pp. 164-170.

doi:10.1097/01.rti.0000117623.02841.e6

[5] S. M. Wilhelm, A. V. Robinson, S. S. Krishnamurthi and H. L. Reynolds, "Evaluations and Management of Incidental Thyroid Nodules in Patients with Another Primary Malignancy,” Surgery, Vol. 142, No. 4, 2007, pp. 581-586, discussion pp. 586-587. doi:10.1016/j.surg.2007.06.033

[6] S. K. Shetty, M. M. Maher, P. F. Hahn, E. F. Halpern and S. L. Aquino, "Significance of Incidental Thyroid Lesions Detected on CT: Correlation among CT, Sonography, and Pathology," American Journal of Roentgenology, Vol. 187, No. 5, 2006, pp. 1349-1356. doi:10.2214/AJR.05.0468 